\title{
Magnetoacoustic wave propagation in off-limb polar regions
}

\author{
E. O'Shea ${ }^{1}$, D. Banerjee ${ }^{2}$, and J. G. Doyle ${ }^{1}$ \\ 1 Armagh Observatory, College Hill, Armagh BT61 9DG, Northern Ireland \\ e-mail: [eos; jgd] @arm.ac.uk \\ 2 Indian Institute of Astrophysics, II Block, Koramangala, Bangalore 560 034, India \\ e-mail: dipu@iiap.res.in
}

Received 23 June 2005 / Accepted 22 February 2006

ABSTRACT

\begin{abstract}
Aims. To find evidence of magnetoacoustic waves in off-limb polar regions.
Methods. Using temporal series data from the Coronal Diagnostic Spectrometer (CDS) on SOHO, we study oscillations found in radiant flux and velocity measurements from transition region and coronal spectral lines. We use Fourier techniques to measure phase delays between flux ("intensity") oscillations and between velocity oscillations of different transition region-corona and corona-corona line pairs. We also measure the phase delays between flux and velocity oscillations $(I-V)$.

Results. The phase delays measured between different line pairs, when plotted over a $-180^{\circ}$ to $+180^{\circ}$ range, line up along diagonal lines corresponding to measurable and fixed time delays. The slopes of these diagonal lines suggest the outward propagation of waves. Using the measured time delays, we estimate propagation speeds for the different line pairs that indicate that the waves producing the observed phase delays are magnetoacoustic waves propagating at speeds close to the sound speed. In addition, we find that the phases occur at fixed integer frequencies of $f / 4\left(90^{\circ}\right)$ and $3 f / 16\left(67.5^{\circ}\right)$, instead of the expected interval of $f\left(360^{\circ}\right)$, indicating that a "Doppler effect" is acting on the waves.

Conclusions. From $I-V$ measurements, we find evidence for fast magnetoacoustic waves to be predominantly present at coronal temperatures, while at transition region temperatures slow magnetoacoustic waves are more common. We find strong evidence for outwardly propagating slow magnetoacostic waves in off-limb polar regions and an indication that these propagating waves are influenced by some form of resonant cavity through which they pass.
\end{abstract}

Key words. Sun: oscillations - Sun: transition region - Sun: atmosphere - Sun: corona

\section{Introduction}

Evidence for waves in the outer atmosphere of the Sun comes from measurements of radiant flux and Doppler velocity oscillations in a range of frequencies, including visible, ultraviolet, $\mathrm{X}$-ray and radio, given off by different solar structures at chromospheric, transition region and coronal temperatures. A number of studies (Ofman et al. 1997; DeForest \& Gurman 1998; Banerjee et al. 2000, 2001a,b) have measured oscillations in plumes, interplumes and coronal holes in the polar regions of the Sun. All of these studies point to the presence of compressional waves, thought to be slow magnetoacoustic waves as found by DeForest \& Gurman (1998). Up to now evidence for the fast magnetoacoustic wave modes in these same regions has been absent, even though recent results by Verwichte et al. (2005) have shown that propagating fast magnetoacostic waves can be present in open magnetic field structures, albeit in this instance, in a post-flare supra-arcade. In this work we look for evidence of propagating magnetoacoustic waves in polar regions of the Sun.

\section{Observations and data reduction}

For these observations we have used the normal incidence spectrometer (NIS), which is one of the components of the Coronal Diagnostic Spectrometer (CDS) on board the Solar and Heliospheric Observatory (SOHO), see Harrison et al. (1995).

The temporal series SER150W sequence was run during November/December 2002 mainly in the Northern polar region, with a single observation in the Southern polar region (see Table 1). During the period of the observations the coronal holes at the poles were ill-defined, particularly so in the
Table 1. SER150W datasets obtained using the $4^{\prime \prime} \times 240^{\prime \prime}$ CDS slit and an exposure time of $60 \mathrm{~s}$ during November/December 2002.

\begin{tabular}{llcl}
\hline \hline Date & Dataset & Pointing $(X, Y)$ & Start/End (UTC) \\
\hline $29 / 11$ & 26348 & 116,1085 & $18: 42 / 21: 32$ \\
$02 / 12$ & 26363 & 54,1070 & $07: 50 / 10: 41$ \\
$06 / 12$ & 26406 & $-1,1070$ & $07: 00 / 09: 51$ \\
$11 / 12$ & 26438 & $-19,1070$ & $16: 29 / 19: 19$ \\
$12 / 12$ & 26447 & 56,1070 & $16: 30 / 19: 20$ \\
$17 / 12$ & 26478 & 59,1071 & $18: 03 / 20: 54$ \\
$18 / 12$ & 26482 & $-1,-1076$ & $06: 58 / 07: 36$ \\
$27 / 12$ & 26542 & 0,1070 & $18: 10 / 21: 00$ \\
\hline
\end{tabular}

Northern polar region. This can be clearly seen in Fig. 1 where the slit locations for the four (representative) CDS datasets, 26348, 26406, 26478 and 26542, are shown over-plotted on EIT $171\left(\log T \approx 1.3 \times 10^{6} \mathrm{~K}\right)$ and EIT $284\left(\log T \approx 2 \times 10^{6} \mathrm{~K}\right)$ images, which were observed at times approximate to those of the CDS datasets. Data were obtained for 11 transition region and coronal lines. However, here we shall only discuss four of these; the transition region line of O v $629.73 \AA\left(\approx 2.5 \times 10^{5} \mathrm{~K}\right)$ and the coronal lines of $\mathrm{Mg} \times 609.79,624.94 \AA\left(\approx 1.25 \times 10^{6} \mathrm{~K}\right)$ and Si XII $520.67 \AA\left(\approx 2.5 \times 10^{6} \mathrm{~K}\right)$. Note that we shall henceforth refer to the lines without the following decimal places, e.g., 629 in place of 629.73.

As reported in Table 1, the exposure time for each of the datasets was $60 \mathrm{~s}$, leading to a cadence of $\approx 68 \mathrm{~s}$ in each case. For each of the 8 datasets listed in Table 1, 150 time frames were obtained in a sit-and-stare study, that is, the CDS slit was left at the same pointing over the whole observation time. The total observation time, therefore, for each dataset is 

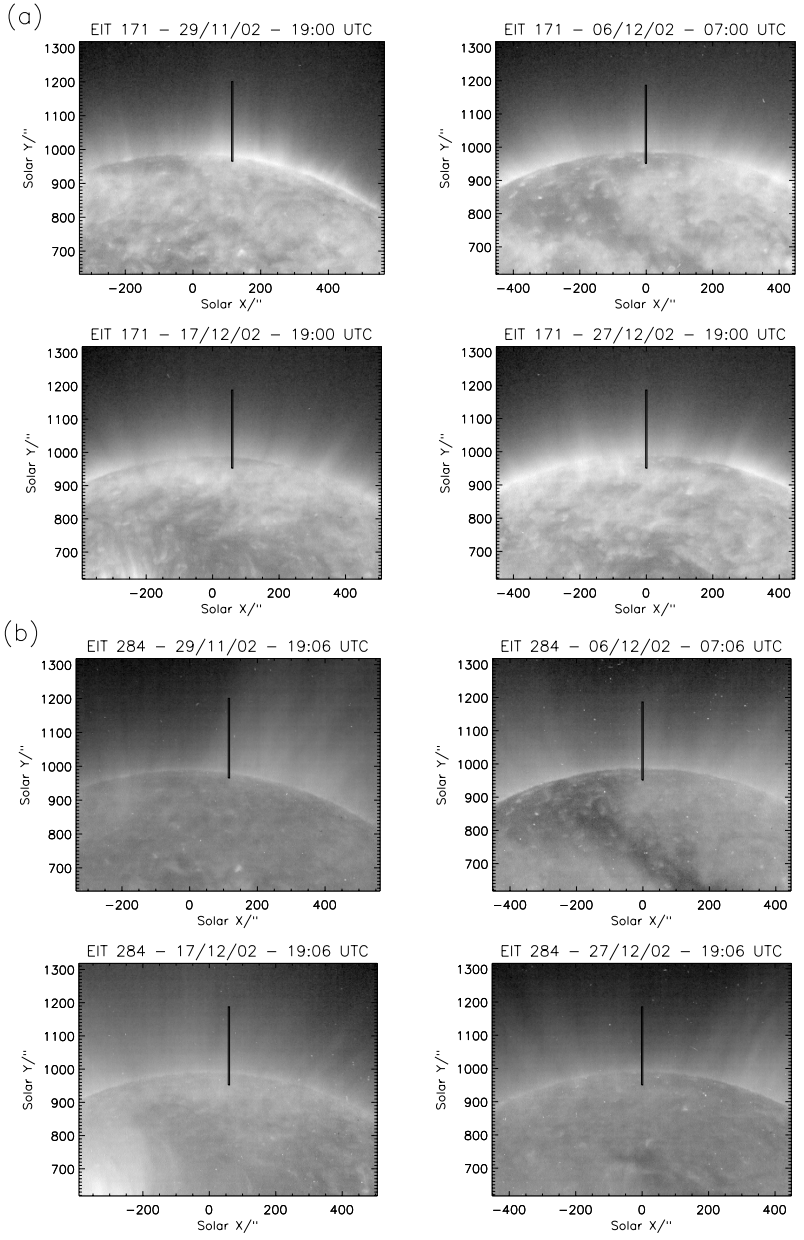

Fig. 1. a) EIT 171 images of the Northern pole of the Sun showing the over-plotted slit positions for the 26348 dataset (top left), the 26406 dataset (top right), the 26478 dataset (bottom left) and the 26542 dataset (bottom right). b) EIT 284 images showing the overplotted slits position for the 26348 dataset (top left), the 26406 dataset (top right), the 26478 dataset (bottom left) and the 26542 dataset (bottom right). Dates and times of the EIT observations are shown in the image titles.

$150 \times 68 \mathrm{~s} \approx 1.02 \times 10^{4} \mathrm{~s} \approx 170 \mathrm{~min}$, the frequency resolution is $\approx 9.80 \times 10^{-2} \mathrm{mHz}$ and the Nyquist frequency is $\approx 7.35 \mathrm{mHz}$. In the following (Fourier) analysis, phase delays for each dataset will be measured at all frequencies up to the Nyquist frequency, at steps dictated by the frequency resolution. As we are measuring above the limb, solar rotation will not have an effect on the minimum measurable frequency of these observations. However, we choose to measure from a minimum frequency of $0.27 \mathrm{mHz}$, indentical to the minimum measurable frequency obtainable from a wavelet analysis (cf. Fig. 3) due to its COI (Torrence \& Compo 1998), as we do not consider it likely that frequencies lower than this are indentifiable oscillations considering the limited length of the observation run.

The data were reduced using the latest versions of the standard CDS routines ${ }^{1}$. Before fitting the lines with a single Gaussian, and in order to increase the signal-to-noise ratio, we binned by 2 along the 143 pixel slit to produce 70 usable pixels $\left(4^{\prime \prime} \times 3.36^{\prime \prime}\right)$. We note that the Point Spread Function (PSF) of CDS has a slightly elliptical form with a FWHM in the NorthSouth direction of $8^{\prime \prime}$ (Pauluhn et al. 1999). Hence, there will be

\footnotetext{
${ }^{1}$ http://solg2.bnsc.rl.ac.uk/software/uguide/ uguide. shtml
}

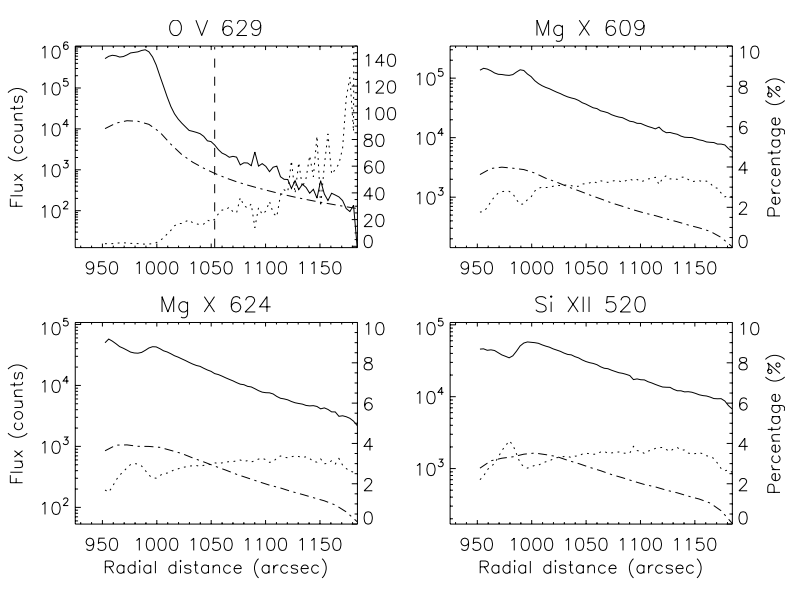

Fig. 2. Measured radiant flux off-limb (solid line), calculated flux due to scattered light (dot-dash line) and the percentage of the total radiant flux due to scattering (dotted line).

some unavoidable overlap between one pixel and the next. This will have the effect that neighbouring pixels may share similar frequencies of oscillation and may also share the same phase differences between line pairs. However, in the context of measurements being taken in up to 70 pixels along the slit in each dataset, and the fact that we are, after all, only interested in the global phase delay characteristics in the larger off-limb area, we feel that this effect can be safely disregarded in our present statistical study.

As these observations were obtained off-limb, the influence of scattered light on the measured line profiles can become nonnegligible. Following the procedure outlined in O'Shea et al. (2005a), we summed over time to produce high signal-to-noise line profiles along the slit to use as the model (the solar brightness distribution) in the light scattering calculations. For locations below $\approx 950^{\prime \prime}$, where we lacked observational data, we used an approximate value for the radial flux distribution calculated from the $1 / \cos \theta$ function, where $\theta$ is the angle between the viewing direction and a perpendicular line normal to the surface. The results of these calculations for the 26363 dataset are shown in Fig. 2 for each of the four lines. Other datasets show almost identical results. For the three coronal lines, $\operatorname{Mg}$ X 609, 624 and Si XII 520, the percentage of the total radiant flux due to scattering can be seen to be no higher than $4 \%$ at all heights. For the transition region O v 629 line, the percentage of the total radiant flux due to scattering can rise to over $100 \%$ at high altitudes offlimb. To account for this problem we limited our analyses with $\mathrm{O} v$ to only those locations along the slit where its summed flux over time had values greater than 4800 , equivalent to an average signal-to-noise value of $\approx 4$ for each of the 150 time frames in the CDS data. For the 26363 dataset shown in Fig. 2, this flux level corresponds to a height of $\approx 1053^{\prime \prime}$ (pixel 30 along the slit) and is indicated by the vertical dashed line in the plot. Locations at heights lower than $\approx 1053^{\prime \prime}$ all have scattered light values of less than $20 \%$. While a scattered light value of $20 \%$ is still quite large we do not consider that it has an important effect on the overall results in this paper due to the use of high significance levels $(>95 \%)$ used in determining the measured phase delays.

\section{Results}

In Fig. 3 we show an example of the type of oscillations measured by us in the off-limb polar region. These oscillations are taken from pixel 11, at a height of 989", in dataset 26363 and 

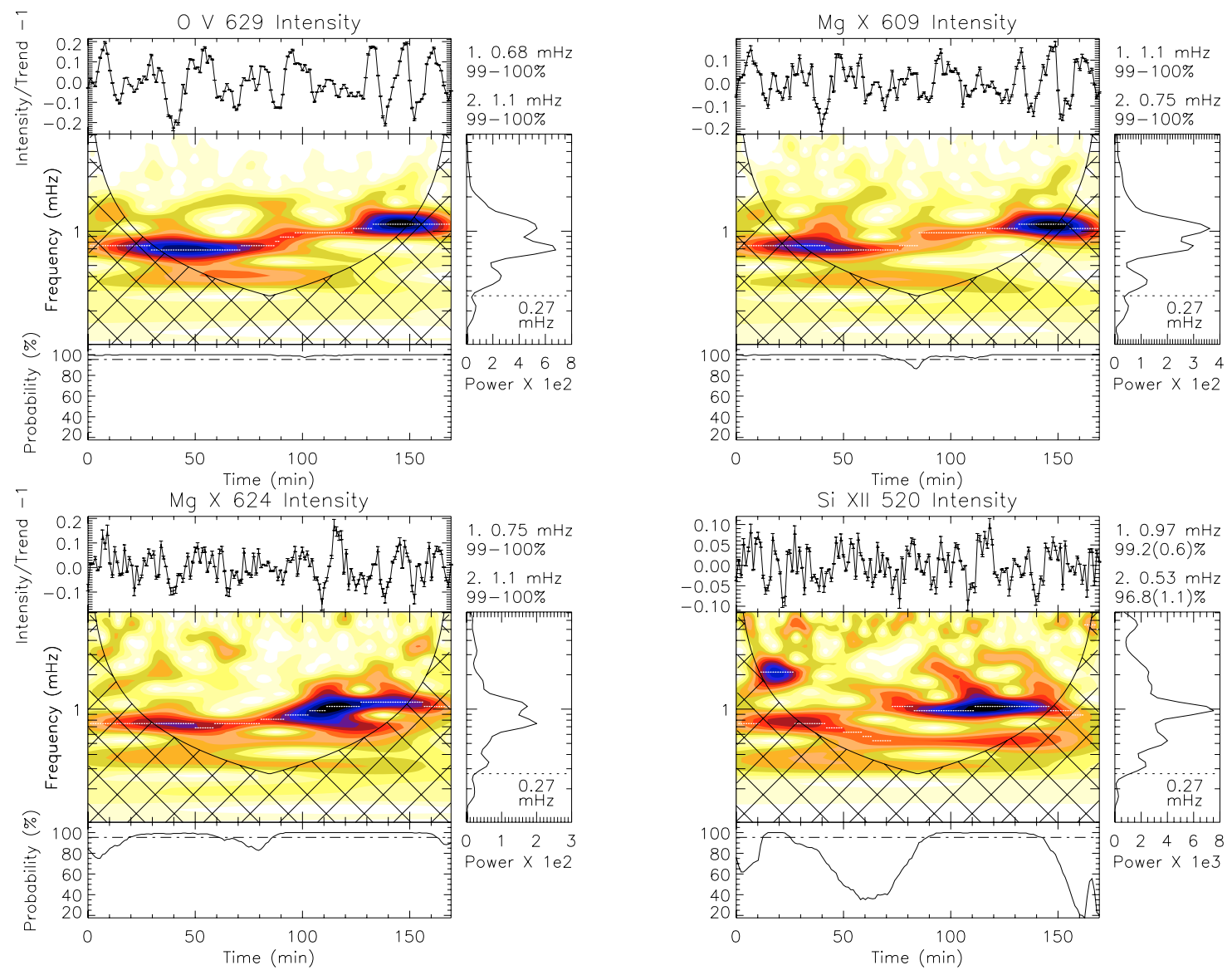

Fig. 3. Wavelet results for the four lines, as labeled. In the top panel is shown the relative (background trend removed) radiant flux, the "intensity", with uncertainties indicated by error bars, in the central panels the colour inverted wavelet power spectrum, in the bottom panel the variation of the probability estimate associated with the maximum power at each time in the wavelet power spectrum (marked with the dotted white lines), and in the right middle panel the global (averaged over time) wavelet power spectrum. Above the global wavelet are printed the frequencies measured from the first and second maxima in the global wavelet spectrum, together with estimates of probability (errors in brackets) that these oscillation are not due to noise. The cross-hatched regions in the wavelet spectrum are locations where estimates of the oscillation frequency become unreliable. This is the so-called cone-of-influence (COI), see Torrence \& Compo (1998). As a result of the COI the minimum measurable frequency is $0.27 \mathrm{mHz}$ (dotted line in the global spectrum plots).

show the variation of the radiant flux as a function of time. Details on the wavelet analysis, which provides information on the temporal dependence of a signal, are described in Torrence \& Compo $(1998)^{2}$. For the convolution with the time series in the wavelet transform we chose the Morlet function as defined in Torrence \& Compo (1998). The oscillations shown in Fig. 3 have had their background trend removed by dividing the original time series by a 25 -point running average (i.e., $\mathrm{a} \approx 28 \mathrm{~min}$ interval). We produce the relative fluxes (the "intensities") shown in the plot by then subtracting a value of one from this trend removed time series. Each of the time series is shown with error bars representing the uncertainties in the fluxes for each of the lines. Above the global wavelet spectrum of each line in Fig. 3 are shown the frequencies measured from the first and second maxima in this global wavelet spectrum, together with estimates of probability that these oscillations are not due to noise. These probability estimates were calculated using the randomisation method as outlined in O'Shea et al. (2001), with 250 permutations. Only those oscillations with a probability $>95 \%$ are considered to be significant. It is clear from these measured frequencies that the different lines share common oscillations, with each of the lines showing the presence of an oscillation at $\approx 1 \mathrm{mHz}$ as either the first or second maximum. The shape of the wavelet power spectrum in the central panels is also similar for each line, showing an oscillation group for the first $\approx 50-70 \mathrm{~min}$ at $\approx 0.8 \mathrm{mHz}$, followed by another oscillation group at $\approx 1 \mathrm{mHz}$ that lasts for the remainder of the observing period. Below the wavelet power spectrum, in the lower panels, we show the variation of the probability estimate, again calculated using the randomisation technique, associated with the maximum power at each time in the wavelet power spectrum (marked with the white lines in the plot).

The shared oscillations between lines separated by large ranges of temperature suggests that there is a link between the cooler transition line of $\mathrm{O} \mathrm{V}$ and the hotter coronal lines of $\mathrm{Mg} \mathrm{X}$ and Si XII. We suggest that the reason for the shared oscillations between these lines is due to waves propagating between the different temperature regions where these lines form.

To investigate whether this is the case we measured the phase delays in flux and also in the line-of-sight (LOS) velocity between different line pairs for each of the measurable pixels along the slit. In this work we follow the treatment used by Athay \& White (1979) in which the calculated phase delays are plotted over the full $-180^{\circ}$ to $+180^{\circ}\left(360^{\circ}\right)$ range and as a function of the measured oscillation frequency. Since the expected phase delay or difference is given by the equation,

\footnotetext{
${ }^{2}$ See http://paos.colorado.edu/research/wavelets/ $\Delta \phi=2 \pi f T$,
} 

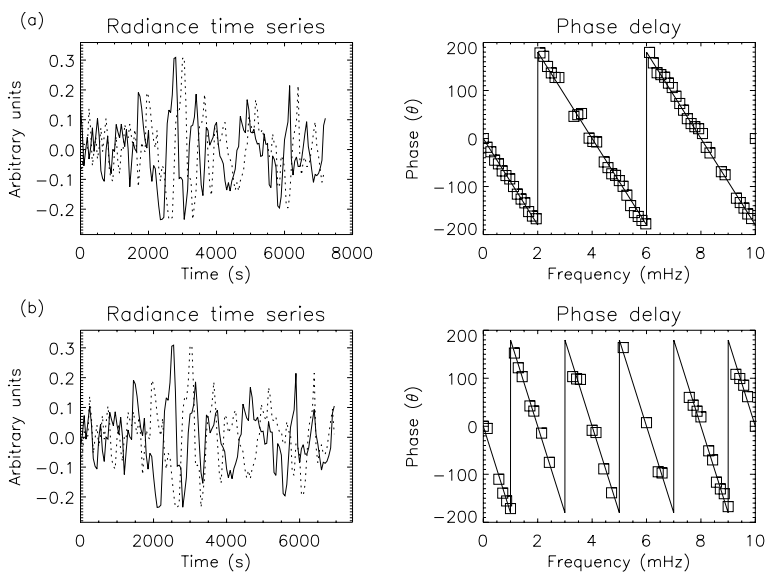

Fig. 4. a) (Left panel) Simulated data showing the presence of a fixed $250 \mathrm{~s}$ time delay between two time series (solid and dotted lines). (Right panel) Calculated phase delay results for the simulated data (square symbols) overplotted with the expected phase delays for a fixed time delay of $250 \mathrm{~s}$ (solid line). b) The same results for a fixed time delay of $500 \mathrm{~s}$.

where $f$ is the frequency and $T$ the time delay in seconds, the phase difference will vary linearly with $f$, and will change by $360^{\circ}$ over frequency intervals of $\Delta f=1 / T$. This will give rise to parallel lines in the $\Delta \phi$ vs. $f$ plots at fixed frequency intervals ( $\Delta f=1 / T)$, corresponding to a fixed time delay $T$.

To show this more clearly, we plot, in Fig. 4, a simulation showing the expected results for two different "observed" datasets, one with a fixed time delay of $250 \mathrm{~s}$ between two "lines" and the other with a fixed time delay of $500 \mathrm{~s}$ between the same two "lines". Both simulated datasets have a cadence of $50 \mathrm{~s}$. In the right-hand panel of Fig. $4 \mathrm{a}$ it can be seen that, for a fixed time delay of $250 \mathrm{~s}$, the calculated phase delays at various frequencies (square symbols) align themselves in parallel linear rows at $4 \mathrm{mHz}$ intervals, as expected. At $1 \mathrm{mHz}$, for example, the phase delay is $-90^{\circ}$, one quarter of the cycle, which is what one would expect for a time delay of $250 \mathrm{~s}$ between two oscillations sharing a $1000 \mathrm{~s}$ period, while at $2 \mathrm{mHz}$ the phase delay is $-180^{\circ}$, one half cycle, again what we would expect for a time delay of $250 \mathrm{~s}$ between two oscillations sharing a $500 \mathrm{~s}$ period. After one half cycle is reached, however, there is a change, as one line changes from lagging the other to leading it, and the phase delays become positive, e.g., for the $2.5 \mathrm{mHz}$ ( $400 \mathrm{~s}$ period) oscillation the half cycle of $200 \mathrm{~s}$ is now less than the fixed time delay of $250 \mathrm{~s}$, producing the result that one line is now leading the other by $150 \mathrm{~s}$ or $135^{\circ}$.

The solid lines in Fig. 4a are the result of plotting the calculated phase delay variation using Eq. (1) with $\Delta f=1 / T(250 \mathrm{~s})=$ $4 \mathrm{mHz}$. As expected, the calculated phase delays show a rapid change over small values of $\Delta f$, and vary by a full $360^{\circ} \mathrm{ev}-$ ery $4 \mathrm{mHz}$. The vertical line segments are simply the result of the rapid flip change from $-180^{\circ}$ to $+180^{\circ}$ every half cycle. Comparing the calculated phase delays (the solid lines) with the data points (the square symbols) it is therefore obvious, even without the benefit of the left-hand panel in Fig. 4a, that one line is delayed relative to the other at a fixed time delay of $250 \mathrm{~s}$. In Fig. 4b the same results can be seen for a time delay of $500 \mathrm{~s}$, where the phase delays now show a rapid change every $2 \mathrm{mHz}$.

As Athay \& White (1979) state in their paper, it is because of this rapid change in $\Delta \phi$ that phase differences can only be determined unambiguously by careful measurements over the full range of observed frequencies. For example, individual

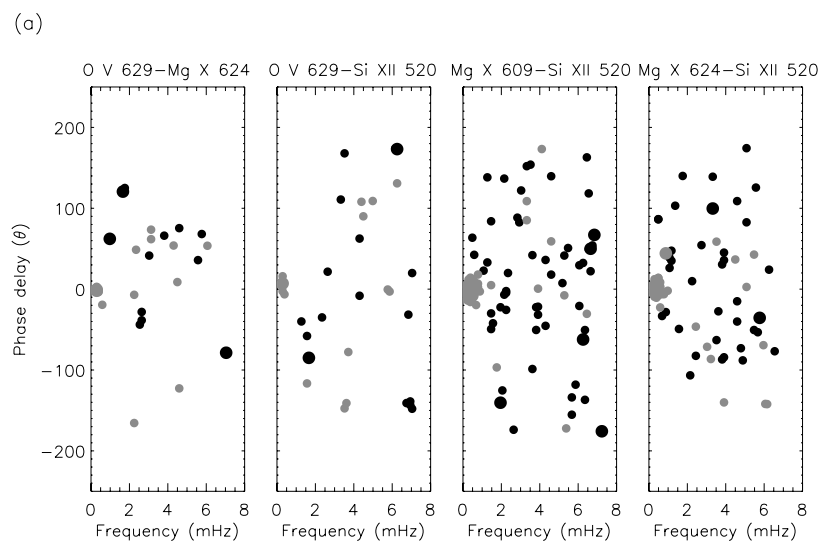

Fig. 5. Dataset 26348 - Phase delay measurements between different line pairs, as labeled, e.g., between $\mathrm{OV}$ and $\mathrm{MgX} 624$ (left panel). Measurements from radiant flux oscillations are shown as the black circle symbols, while L.O.S. velocity oscillations are shown as the grey circle symbols. Phase delays were measured at the $95 \%$ and $99 \%$ significance levels. Phase delays at the $99 \%$ significance level (if present) are indicated by the slightly larger symbols for both radiant flux and velocity.

measurements taken at widely separated frequencies in Fig. 4 would lead to widely varying estimates of phase delay. A measurement of phase delay at the frequency of $2 \mathrm{mHz}$ in Fig. $4 \mathrm{a}$ would suggest a value of $-180^{\circ}$, while a measurement at $4 \mathrm{mHz}$ would suggest a value of $0^{\circ}$. It is therefore only within the context of full measurements over the total frequency range that we can begin to see evidence for a single fixed time delay between oscillations.

We note here that for different time delays the slope or angle of the linear variations (with respect to the horizontal) changes. As the time delay becomes longer the slope becomes more acute. This can be easily seen by comparing the phase delay plots in Figs. $4 \mathrm{a}$ and $4 \mathrm{~b}$. Therefore, by measuring the slope of the linearly arranged phase delays we have a simple method of obtaining estimates of the time delay.

In summary, the technique that will be used here involves plotting measured phase delays as a function of all observed frequencies and then overplotting sloping lines, corresponding to fixed time delays, that are as close a fit as possible to these measured phase delays. In this way, fixed time delays between oscillations at different temperatures may be accurately measured. We point out that this technique of measuring phase delays offers us a powerful tool to measure time delays between oscillations in lines of different temperatures, and hence to infer information on the wave modes producing these oscillations.

In Fig. 5, we show the results of phase delay measurements for one of the seven datasets observed in the Northern polar region (see Table 1) and corresponding to all measurable pixels (up to a maximum of 70) in that dataset. Phase delays in these plots were calculated using confidence levels of $95 \%$ and $99 \%$, that is, only those measured phase values with a squared coherency spectral estimate above these levels were selected. The phase delays measured at the $99 \%$ significance level are shown as the slightly larger circle symbols. Typical uncertainties in these phase delays measurements are shown as the error bars in the later combined plot shown in Fig. 6. To calculate the phase delays we used standard Fourier analysis techniques, following closely the "recipe" given in Appendix A of Doyle et al. (1999). We chose the Tukey window as our window function in that analysis. 

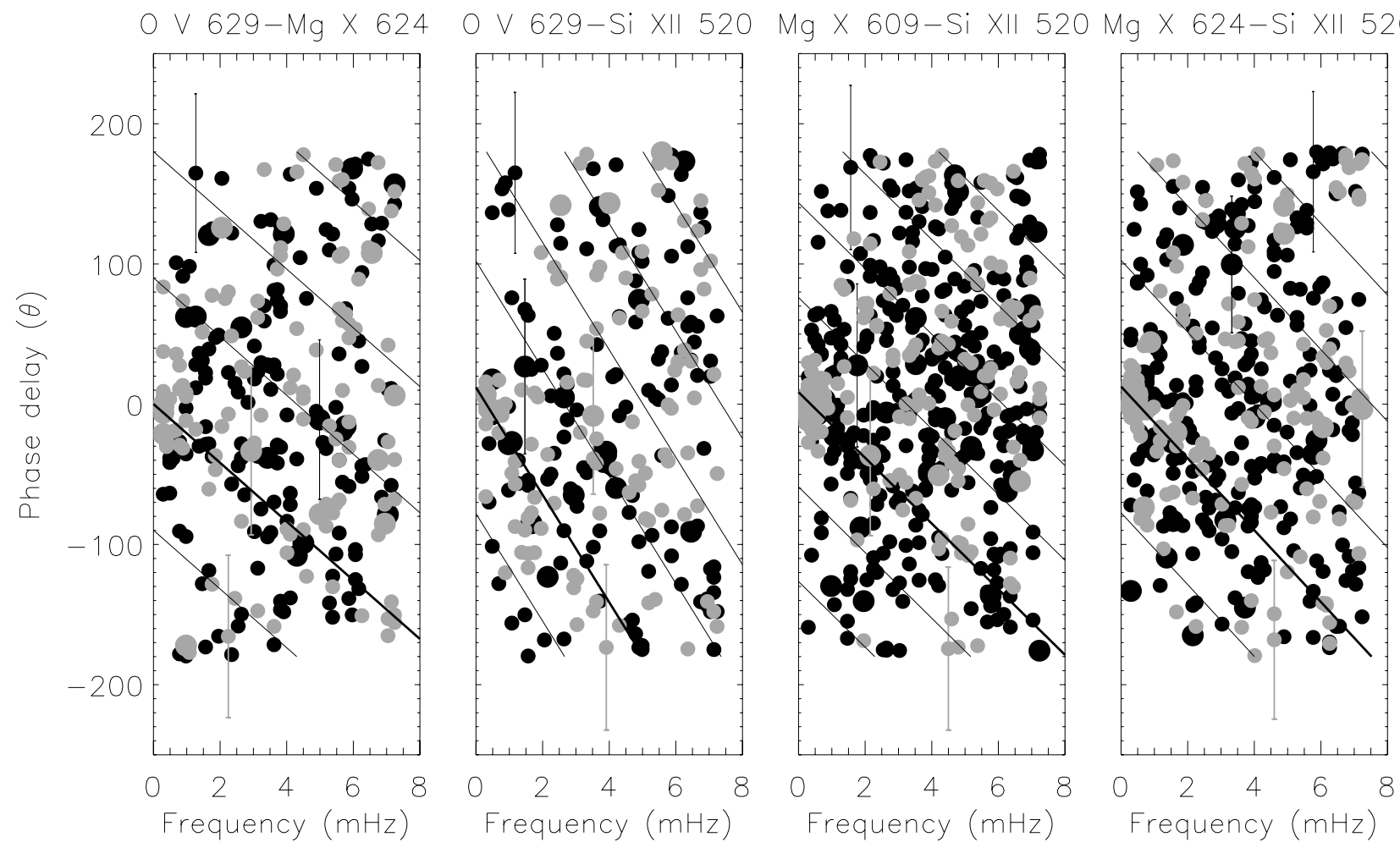

Fig. 6. Phase delays measured between the oscillations in the different line pairs, as labeled, e.g., between Ov and Mg X 624 (left panel). Phase delays from radiant flux oscillations are shown as the black circle symbols, while phase delays from L.O.S. velocity oscillations are shown as the grey circle symbols. Phase delays were measured at the $95 \%$ and $99 \%$ significance levels. Phase delays at the $99 \%$ significance level are indicated by the slightly larger symbols. Average uncertainties in the $95 \%$ and $99 \%$ phase delay estimates are shown by the representative error bars in each plot. Over-plotted on this plot are lines corresponding to fixed time delays.

Note that while we used wavelet techniques to display the results in Fig. 3, we prefer to use Fourier techniques to perform the statistical study carried out in this paper. Wavelet techniques have the advantage of showing the temporal extent of any oscillating group and, in fact, wavelets have recently been used to measure phase delays (Bloomfield et al. 2004). However, in this statistical study we are only interested in the presence of phase delays and not the duration over which these phase delays are present, and so we prefer to use the more tried and trusted methods of Fourier phase delay calculations.

From Fig. 5, it can be seen that phase delays are present at a range of frequencies and at varying phase delay values in each of the datasets. While phase delays are found between $-180^{\circ}$ and $180^{\circ}$ in each of the plots, little evidence for a linear pattern at a fixed frequency spacing (as in Fig. 4) can be discerned, at least by eye. We suggest that this is due to the limited number of measurable pixels (a maximum of 70) along the slit, which means that there are an insufficient number of significant points in intensity and velocity to build up a recognisable linear pattern.

To counter this problem and to show the improving influence of statistics, we show, in Fig. 6, the result of combining the results from all seven Northern polar region datasets. We believe that this is justified as all of these datasets were obtained in a relatively reduced area at the North pole of the Sun over a period when the conditions at this location were relatively unchanging, i.e., the polar coronal hole remained ill-defined during this whole period. We note, in addition, that plume locations in polar regions can be remarkably stable, showing a re-occurrence of brightenings in the same site over periods of up to two weeks (DeForest 1998). We cannot say from our observations whether the oscillations measured are originating from plumes or not.
However, the stability of plumes in polar regions over a two week period strongly suggests that the magnetic topology of polar regions in general is relatively stable on timescales of weeks. Therefore, it is also likely to be relatively constant over the period of our observations ( $\approx 4$ weeks). In light of this, we do not think that combining these seven datasets is unrealistic, in order to build up a more coherent picture of the typical phase delays present in the polar region being observed. We believe, furthermore, that the eventual results of our analysis bear out this assumption.

In Fig. 6 it can be seen, that for the line pairs O v 629-Mg X 624, O v 629-Si XII 520, Mg X 609-Si XII 520 and $\mathrm{Mg}$ X 624-Si XII 520, the measured phase delays for flux and velocity appear to lie, at least to a rough approximation, along a number of spaced "lines" that are inclined relative to the horizontal. These "lines", it will be admitted, are barely discernible by eye, but it will be granted that there is some clumping of phase delays taking place, at least. This clumping of phase delays in a roughly linear pattern is what we would expect if there was a fixed time delay between the oscillations in each line pair. The variation and spread of the phase delay values can be accounted for by the relatively large uncertainties in the calculated phase delays plus the relatively small data sample used to produce these statistics. Note that the significance of the sloping solid black lines in this plot will be explained in the following passages.

We note here that the third plot for Mg X 609-Si XII 520 has more points than the other three plots in this figure. That this plot has more points than the plots of O V 629-Mg X 624 and O V 629-Si XII 520 should not be too surprising. In Sect. 2, we state that locations above 1053" (pixel 30) in dataset 26363 
are not considered due to the effects of scattered light. All other datasets are similarly affected at similar altitudes above the limb and, hence, at similar locations along the slit. Hence, due to the reduced number of pixels available in which phase measurements can be made, it is natural that there will be less points in the plots of OV 629-Mg X 624 and OV 629-Si XII than in the plots of $\mathrm{MgX}$ 609-Si XII 520 and MgX 624-Si XII 520, which make use of the full 70 pixels along the slit. There is, however, still the question of why the Mg X 609-Si XII 520 plot has more points than the Mg X 624-Si XII 520 plot. This can be explained by the fact that the $\mathrm{MgX} 609$ line is one of the strongest lines in the CDS wavelength range, stronger than the Mg X 624 line. For a line pair like Mg X 609-Si XII 520 to show a significant phase delay at a particular frequency, the squared coherency spectral estimate obtained from these lines must be above $95 \%$. The chances of this occurring are enhanced if the lines in question are strong and so have a good signal-to-noise ratio. The stronger the lines, the lower the relative noise and so the higher the squared coherency obtained from any measured oscillations. Another reason for the increased number of points in the Mg X 609-Si XII 520 line pair may be due to the fact that the $\mathrm{Mg} \mathrm{X}$ line is blended with transition region lines of O III and O IV at $609.70 \AA$ and $609.83 \AA$, respectively. This may lead to additional phase measurements in flux and velocity between these O III and O IV blends and Si XII in addition to the expected measurements between $\mathrm{Mg} \mathrm{X}$ and Si XII. Off-limb this should not be too much of a problem due to the weakness of the O III and O IV lines in these areas. The blending of the Mg X 609 line with $\mathrm{O}$ III and O IV lines is, however, the reason why we do not present phase delays for the line pair O V 629-Mg X 609. The close proximity in temperature of the $\mathrm{OV} 629$ line to the lines of O III and O IV, that form the blend with Mg X 609, leads to a concentration of phase delay measurements around the zero point of phase when measuring the O V 629-Mg X 609 line pair.

From the discussion above, in relation to Fig. 4, it is known that different time delays should lead to "lines" with different slopes in plots of phase delay vs. frequency. To accurately determine the time delays from the slopes of the "lines" in Fig. 6 we proceed as follows: (1) a rough estimate is first made of the appropriate time delay for each line pair. Using Eq. (1) and this time delay a straight line is drawn through the data. This estimate of the delay can be obtained by trial and error, e.g., by trying different time delay estimates until one produces a line that is a reasonable "fit" to the data; (2) using this straight line "fit", and proceeding along the fitted line with increasing frequency, we choose only those datapoints lying within $\sigma / 2$ of the line at each frequency location, where $\sigma$ is the average uncertainty in the calculated phase delay for all radiant flux and velocity datapoints; (3) taking these chosen points and fitting them with a 1 st order polynomial we are then able to accurately measure the slope $(2 \pi T)$ and, hence, the time delay for each line pair; (4) using this measured time delay and Eq. (1), we then repeat the process in an iterative loop (starting at step 2 above) until such time as the time delay converges to a consistent value. The result of this procedure and the fitted lines are shown in Fig. 7 for each of the line pairs. In the fit with the 1st order polynomial the individual points of the fit are weighted with their different uncertainty (error) estimates. From the treatment described above we obtain time delay values of $58 \pm 7 \mathrm{~s}$ ( $f \approx 17 \mathrm{mHz}$ ) for O v 629-Mg X 624 (left panel of Fig. 6), $106 \pm 14 \mathrm{~s}(f \approx 9.43 \mathrm{mHz})$ for O v 629-Si XII 520 (middle left panel), $65 \pm 5 \mathrm{~s}(f \approx 15 \mathrm{mHz}$ ) for Mg X 609-Si XII 520 (middle right panel) and $71 \pm 6 \mathrm{~s}(f \approx 14 \mathrm{mHz})$ for $\mathrm{Mg}$ X 624-Si XII 520 (right panel).

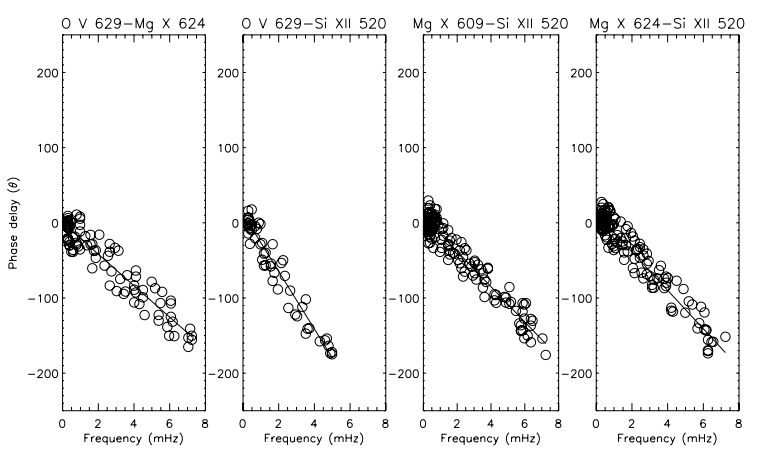

Fig. 7. Fitted lines to the measured phase delays. Values within $\sigma / 2$ of the solid line are included in the fit.
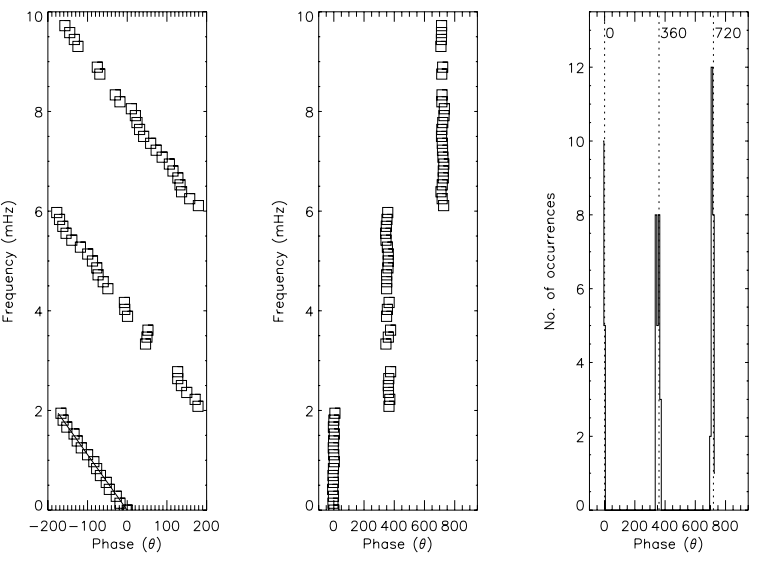

Fig. 8. (Left panel) Simulated phase measurements at a fixed $250 \mathrm{~s}$ time delay. The datapoints passing through the zero of phase have been fitted with a line. (Middle panel) The result of shifting the phase measurements to the vertical using the slope of the fitted line. (Right panel) A histogram of the results in the middle panel showing the separation of the datapoints at $360^{\circ}$ intervals as expected.

Now, looking at Fig. 6 again, it is clear that there are scattered datapoints present in discernible linear clumps between $-180^{\circ}$ and $+180^{\circ}$. From Eq. (1) and the discussion relating to Fig. 4, we would expect the spacing between these clumps to be $1 / T$, where $T=$ time delay. Looking at the plot in Fig. 6 for O v 629-Mg X 624 (left panel), it is clear that the spacing is much less than the time delay of $58 \mathrm{~s}$ (a spacing of $\approx 17 \mathrm{mHz}$ ) would suggest. That is, based on the discussion relating to Fig. 4 , we would expect the distance between any discernible clumps to be $360^{\circ}$ over a frequency spacing of $\approx 17 \mathrm{mHz}$. We clearly do not see that in our plot where the distance between the clumps is some fraction of $360^{\circ}$. We can see that the same is true of all four line pairs measured.

To investigate this, and in order to accurately measure the spacing between different clumps of phase points, we use the slopes of the fitted lines shown in Fig. 7 in order to shift all the datapoints in Fig. 6 up to the horizontal. That is, we shift up (or down) all the datapoints at each frequency by the difference between the phase value of the fitted line at that frequency and the zero value itself.

To illustrate this technique, we show in Fig. 8, the result of performing the same procedure on the simulated phase data plotted in Fig. 4a. In the left panel of Fig. 8 are shown the original phase measurements. Note that here we have plotted the phases on the $x$-axis. The datapoints passing through the zero of phase have been fitted with a line, as in Fig. 7. In the middle plot is shown the result of using the slope of this fitted line to shift all 

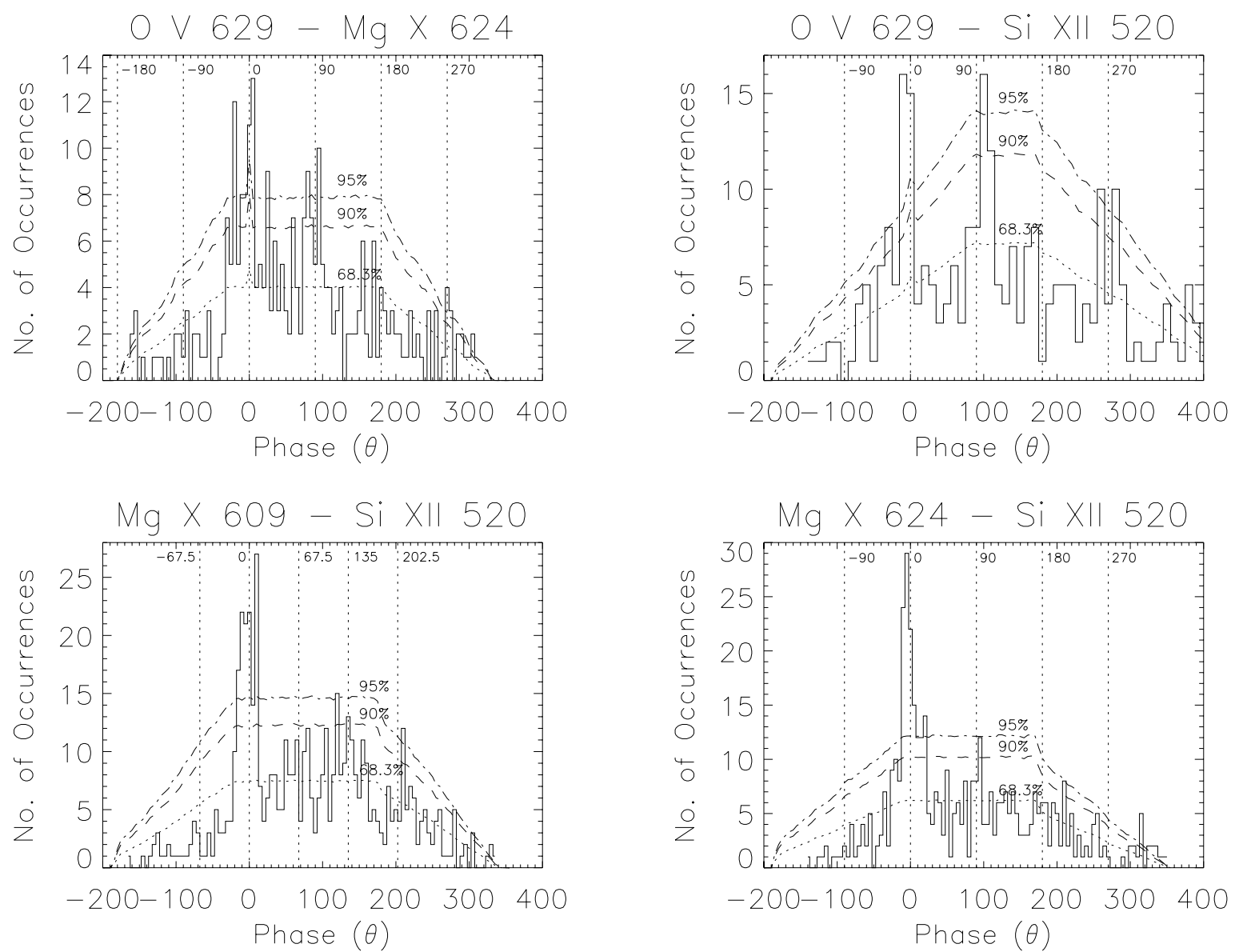

Fig. 9. Histograms showing the distribution of phase delay measurements as a function of frequency. Dotted vertical lines indicate phase intervals of $90^{\circ}$ and $67.5^{\circ}$ (for the $\mathrm{Mg}$ X 609-Si XII 520 histogram, bottom left). Overplotted on the histograms as dotted, dashed and dot-dashed horizontal lines are the results of Monte Carlo simulations with 5000 permutations. The dotted lines shows the expected noise distribution, i.e., the $1 \sigma, 68.3 \%$ confidence level; the dashed lines the $90 \%$ confidence level, i.e., the $\approx 1.6 \sigma$ level; and the dot-dashed lines the $95 \%$ confidence level, i.e., the $\approx 2 \sigma$ level.

the datapoints over to the vertical (not to the horizontal as the phase here, unlike Fig, 7, is plotted on the $x$-axis). Finally, in the right panel is shown a histogram constructed from the results in the middle panel. It is instantly clear from this histogram that there is a separation of $360^{\circ}$ between the sloping lines, as expected. Producing histograms in this way allows us an easy way to accurately measure the spacing present in the phase plots shown in Fig. 6.

By carrying out this procedure, and shifting all the datapoints in Fig. 6 to the horizontal, histograms of the phase distribution as a function of frequency were produced. We show the results of this in Fig. 9. All the histograms are produced with a bin size of $5^{\circ}$ except for the O V 629-Si XII 520 histogram, which is produced with a bin size of $10^{\circ}$. The spacing chosen for the histogram bin is directly dependent on the error in the $y$-intercept of the fitted lines shown in Fig. 7.

In Fig. 9, vertical dotted lines spaced at $90^{\circ}$ intervals are overplotted on the histograms of O V 629-Mg X 624 (top left), O V 629-Si XII 520 (top right) and Mg X 624-Si XII 520 (bottom right). For the $\mathrm{Mg}$ X $609-\mathrm{Si}$ XII 520 histogram the spacing of the vertical dotted lines is every $67.5^{\circ}$. Also overplotted on these plots are three horizontal lines (dotted, dashed and dot-dashed) showing confidence levels at $68.3 \%(1 \sigma), 90 \%(\approx 1.6 \sigma)$ and $95 \%$ $(\approx 2 \sigma)$, respectively, calculated from a Monte Carlo or randomisation test. To produce these confidence levels the orignal phase measurements in Fig. 6 were first randomised by shuffling the locations of the measurements between -180 and $180^{\circ}$. This was done by using the random number generator, RANDOMU, of the IDL data analysis software. Using the randomised phase plots thus produced, we followed the same method of creating histograms as for the real data points. That is, the slopes of the fitted lines in Fig. 7 were used to shift all of the randomised datapoints to the horizontal. From these shifted datapoints a new "randomised" histogram was then created. Carrying out this same procedure for 5000 permutations allowed us to calculate the expected noise distribution, i.e., the $1 \sigma$ (68.3\% confidence) level, by averaging all of the resulting randomised histograms and, hence, to also calculate the other confidence levels at $\approx 1.6 \sigma$ $(90 \%)$ and $\approx 2 \sigma(95 \%)$.

We note that the randomisation test used here is based on the (null) assumption that, if there is, in fact, no linear structure present in the phase measurements in Fig. 6, then the measured phase values are independent of their location along the Y-axis (i.e., their location between $-180^{\circ}$ and $180^{\circ}$ ). For example, if they are truly random then the phases $P_{y 1}, P_{y 2}, \ldots P_{y n}$ are just as likely to have occurred in any other order, $P_{r(y 1)}$, $P_{r(y 2)}, \ldots P_{r(y n)}$, where $n$ is the total number of phase locations and $r(y 1), r\left(y^{2}\right), \ldots r(y n)$ is a random permutation of the subscripts $y 1, y 2, \ldots y n$. See Linnell Nemec \& Nemec (1985) and O'Shea et al. (2001) for its use in time series analysis.

If we look at the histogram for the O v 629-Mg X 624 line pair (Fig. 9, top left), we can see that the two main peaks in 
the histogram at $0^{\circ}$ and $\approx 90^{\circ}$ both reach the $95 \%$ confidence level. There is an obvious spread in the width of the histograms due to the uncertainties in the measured phases. The average uncertainties in phase are shown as the error bars on some of the flux and velocity measurements in Fig. 6. These uncertainties of $\approx 60^{\circ}$ can account for the spread of phase around $0^{\circ}$ and $90^{\circ}$, e.g., producing the smaller significant peaks above the $95 \%$ level at $-20^{\circ}$ and $25^{\circ}$ as values from $0^{\circ}$ are spread into neighbouring histogram bins. We note that another smaller peak at $270^{\circ}$ also reaches the $95 \%$ confidence level while those at $\approx-90^{\circ}$ and $\approx 180^{\circ}$ are either slightly above or at the level of noise (the $1 \sigma$ level) and so are not statistically significant.

So what can we conclude from this first histogram? Firstly, we can be confident that the peaks above the $95 \%$ confidence level in the $\mathrm{O} v 629-\mathrm{Mg} \mathrm{X} 624$ histogram are not likely to be due to noise and so are statistically significant. Secondly, notwithstanding the fact that some of the peaks in the histogram (e.g., at $\approx-90^{\circ}$ and $\approx 180^{\circ}$ ) are not statistically significant, due we suggest to a lack of statistical points in our plots, we can now ascertain from the O v 629-Mg X 624 histogram that the barely discernible (by eye) linear clumping in Fig. 6 is, in fact, real. This is clearly the case at the $0^{\circ}, 90^{\circ}$ and $270^{\circ}$ phase locations in Fig. 9.

Similar results may be seen in the histograms of the other line pairs examined. For the O V 629-Si XII 520 line pair (Fig. 9, top right), we can see that the peaks at $0^{\circ}, \approx 90^{\circ}$ and $\approx 270^{\circ}$ reach the $95 \%$ confidence level, while the "peaks" at $\approx-90^{\circ}$ and $\approx 180^{\circ}$ (i.e., $170^{\circ}$ ) are either non-existent or only reach the noise level. Again, the spread of the phases which produces, for example, the significant peaks above the $95 \%$ confidence level at $100^{\circ}$ and at $260^{\circ}$ and $280^{\circ}$, which we refer to above as the $\approx 90^{\circ}$ and $\approx 270^{\circ}$ peaks, can be explained by uncertainties due to noise in the radiant flux and velocity phase measurements.

For the Mg X 609-Si XII 520 line pair (Fig. 9, bottom left), the peaks at $0^{\circ}$ and $\approx 202.5^{\circ}$ are above the $95 \%$ confidence level while the one at $135^{\circ}$ reaches the $90 \%$ level. If we accept that the peak at $120^{\circ}$ is part of a wider and spread out peak centred at $135^{\circ}$, then we can say that the $135^{\circ}$ peak also reaches the $95 \%$ confidence level. This is valid considering the large uncertainties of $\approx 60^{\circ}$ in the phase measurements. The peak at $\approx 67.5^{\circ}$ is some way above the level expected for noise, though without reaching even the $90 \%$ level, while the peak at $\approx-67.5 \%$ is below the level expected even for noise. From these results, we can be confident of the $67.5^{\circ}$ linear spacing of the Mg X 609-Si XII 520 line pair at, at least, the $90 \%$ confidence level at three locations; $0^{\circ}, 135^{\circ}$ and $202.5^{\circ}$.

For the Mg X 624-Si XII 520 line pair (Fig. 9, bottom right), the peaks at $0^{\circ}$ and $\approx 90^{\circ}$ are present at or above the $95 \%$ confidence level. All other peaks in the histogram are at the level of noise.

So what can we say from all the histograms shown here? It is clear from the results of the O V 629-MgX 624, O V 629-Si XII 520 and Mg X 624-Si XII 520 histograms that there is a statistically measurable tendency (at the $90 \%$ and $95 \%$ levels) for the measured phase delays to line up along lines with spacings of $90^{\circ}$. In the case of the Mg X 609-Si XII 520 histogram, the statistical evidence is for lines at $67.5^{\circ}$ spacings. It is not clear why this line pair should show different results from the Mg X 624-Si XII 520 line pair when both Mg X lines are formed at the same temperature. It may be that the $\mathrm{Mg} \mathrm{X} 609$ line is affected by blends of O III and O IV lines at 609.70 and $609.83 \AA$, respectively. For all of these histograms, we consider it very unlikely that the observed distributions with these spacings could have occurred solely by chance. We note that the fact that most
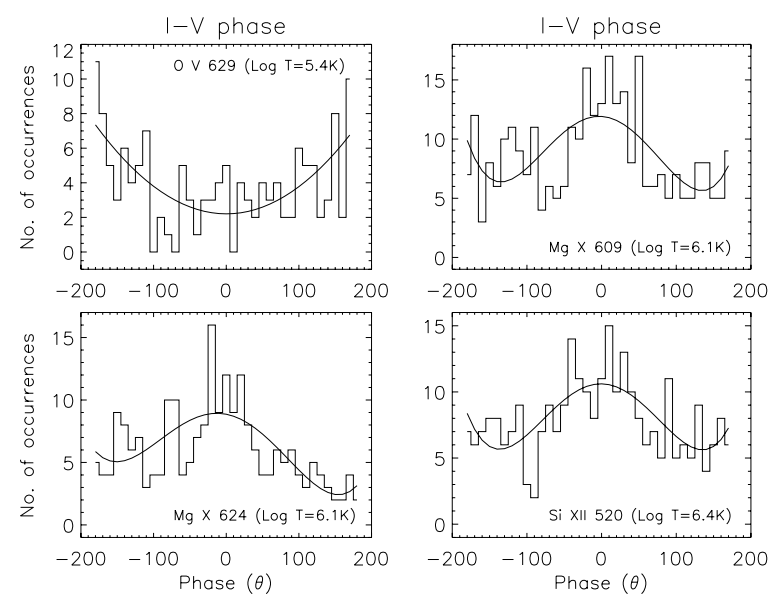

Fig. 10. Flux-velocity $(I-V)$ phase delay plots for each of the lines, as labeled. Over-plotted on each histogram is a polynomial, 2nd order for $\mathrm{OV}$ and 4th order for the three coronal lines.

phases are clustered around $0^{\circ}$ and $90^{\circ}$ is likely to be due solely to the limited statistics used in this work. A longer and more complete statistical study is likely to find that the phases are distributed more evenly at other phase angles.

What do these intervals of $90^{\circ}$ and $67.5^{\circ}$ mean? If we return to Eq. (1), we can see that the results found here can be interpreted as;

$\Delta \phi=2 \pi(f \pm n \Delta f) T$

where $n$ is the order of the frequency shift, i.e., 0,1,2, etc., and $\Delta f$ is $f / 4\left(90^{\circ}\right)$ for the $\mathrm{O} v$ 629-Mg X 624, O v 629-Si XII 520 and the MgX 624-Si XII 520 line pairs and $3 f / 16\left(67.5^{\circ}\right)$ for the Mg X 609-Si XII 520 line pair. This suggests that an additional factor is producing a "Doppler effect" upon the (assumed) waves causing the oscillations, producing positive and negative frequency shifts. This additional effect results in a shift in the observed frequencies by multiples of $f / 4$ and $3 f / 16$, as we have seen. The presence of phase delays at these fixed integer frequencies (i.e., $n=0,1,2$, etc.) suggests that the observed oscillations, and, therefore, the waves producing them, are being influenced by some form of resonant cavity through which the waves pass.

In an homogenous corona, regions of low Alfvén speed (essentially regions of high density, e.g., polar plumes, spicules, etc.) can act as wave ducts, trapping fast magnetoacoustic wave modes (Roberts et al. 1984). We note also that Sterling \& Hollweg (1984) show that spicules can act as resonant cavities for Alfvén waves. We further note that the observations discussed here were obtained in a region where the presence of macrospicules is considered likely (O'Shea et al. 2005b).

If we plot lines on the plots of phase delay vs. frequency in Fig. 6, at slopes corresponding to the measured time delays and at separations of $90^{\circ}$ and $67.5^{\circ}$ (in the case of the Mg X 609-Si XII 520 line pair) we finally get the result shown in this figure. The slightly thicker black line shows the fit used to calculate the time delays, cf. Fig. 7. On a final note, we point out that from the orientation of the slopes in this figure the higher temperature lines are lagging the cooler temperature lines. If the slopes of the lines was reversed the opposite would be the case. This suggests wave propagation from lower to higher temperatures.

What can we say of the waves that are causing these phase delays? In Fig. 10 we plot histograms of the phase delays measured between flux and L.O.S. velocity measurements ( $I-V$ plots). Over-plotted on these histograms are fitted 

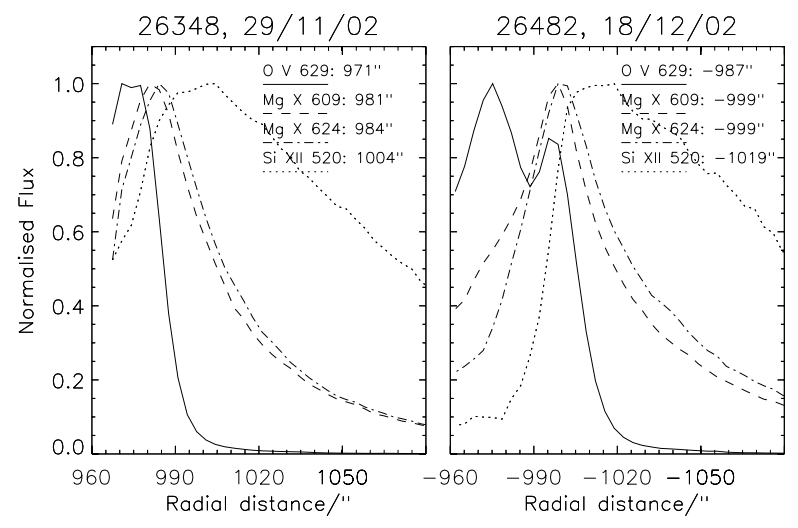

Fig. 11. Normalised flux distribution with height for the 26348 dataset (left panel) and the 26482 dataset (right panel). The locations of maximum brightness for each line are printed in the plots.

polynomials ( 2 nd order for $\mathrm{O} \mathrm{v}$ and 4 th order for the three coronal lines) indicating the overall shape of the distribution. For the $\mathrm{O} v$ line it can be seen that the majority of oscillations in this line possess an $I-V$ phase delay of $\pm 180^{\circ}$, suggesting a majority of propagating sound-like (longitudinal) waves at the temperature of this line. This can be understood if we accept that the majority of the waves at the poles are propagating at almost right angles to our line-of-sight. For longitudinal waves in such an orientation, where the observer sees them side-on, we might expect the location of maximum intensity to also be the locations of the minimum velocity, i.e., at the "crests" of these longitudinal waves. For the coronal lines of $\mathrm{Mg} \mathrm{X}$ and Si XII we see the reverse, with a majority of the oscillations having a phase delay of $0^{\circ}$, suggesting a majority of more Alfvén-like (transverse) waves at these coronal temperatures. Again this can be understood if we accept that we are observing the waves side-on. For a transverse wave in such an orientation the maximum velocity will coincide with the maximum intensity. We know that it is unlikely that pure sound waves can travel up to the transition region and coronal heights under investigation here, and that Alfvén waves would not produce oscillations in the radiant flux. With this in mind, the more sound-like (longitudinal) waves found predominantly in $\mathrm{OV}$ are likely to be slow magnetoacoustic waves, while the more Alfvén-like (transverse) waves found predominantly in the coronal lines are fast magnetoacoustic waves. While the plots in Fig. 10 indicate the majority of waves present at each temperature, they do not help us in determining the exact nature of the waves producing the phase delays in Fig. 6.

In order to determine this, we will need to estimate the propagation speeds of the waves. We already know the time delays between the the different line pairs, e.g., O v 629-Mg X 624, etc., but to calculate the propagation speeds we also need to know the formation heights of the lines in each line pair. To find the formation heights, we plot, in Fig. 11, for datasets 26348 and 26482 (South pole), the summed flux over time for different radial distances along the slit at the limb. The flux for the different lines has been normalised in order to plot them together, and interpolated over intervals of 1 arcsec using the INTERPOL function in IDL. The datasets 26348 and 26482 have been chosen as they are the only two datasets in this study that do not contain significant dynamic activity between the heights of $\approx 980-1000^{\prime \prime}$, of the type described in O'Shea et al. (2005b). The dynamic activity at these heights, and the corresponding brightenings associated with it, has the effect of masking the true location of the limb as inferred from limb brightening measurements. From Fig. 11, we can see that the different lines have different
Table 2. Formation height differences (in arcsec) estimated from limb brightenings. $1^{\prime \prime} \approx 715 \mathrm{~km}$ at the SOHO L1 position.

\begin{tabular}{lccl}
\hline \hline & 26348 & 26482 & Average \\
\hline O v 629-Mg X 624 & 13 & 12 & $12.5(8938 \mathrm{~km})$ \\
O v 629-Si XII 520 & 33 & 32 & $32.5(23238 \mathrm{~km})$ \\
Mg X 609-Si XII 520 & 23 & 20 & $21.5(15373 \mathrm{~km})$ \\
Mg X 624-Si XII 520 & 20 & 20 & $20(14300 \mathrm{~km})$ \\
\hline
\end{tabular}

maximum brightnesses at the limb, reflecting their different formation heights. The exact locations of maximum brightness in each line are printed in the plots. Note that, for the $\mathrm{OV}$ line of 26482, we have estimated the average formation height to be at $-987^{\prime \prime}$, using a weighted average between the two peaks at -975 and $-995^{\prime \prime}$ based on their respective values of flux. We do not believe that the larger peak at $-975^{\prime \prime}$ marks the formation height of $\mathrm{O} v$ due to the presence of the second smaller peak and the tail out to higher altitudes off-limb.

In Table 2 we show the height differences between the different line pairs plotted in Fig. 11, together with the average values from the two datasets. Knowing the time delay between these pairs of lines allows us a simple way to estimate the propagation speeds of the waves in question. For the Ov 629-Mg X 624 line pair the measured time delay was $58 \pm 7 \mathrm{~s}$, which taken with the average height difference between these lines shown in Table 2 points to a wave travelling at a speed of $154 \pm 18 \mathrm{~km} \mathrm{~s}^{-1}$. Similarly, the time delay of $106 \pm 14$ s between OV 629-Si XII 520 and the average height difference points to a wave travelling at a speed of $218 \pm 28 \mathrm{~km} \mathrm{~s}^{-1}$. For Mg X 609-Si XII 520 the time delay of $65 \pm 5 \mathrm{~s}$ points to a wave speed of $236 \pm 19 \mathrm{~km} \mathrm{~s}^{-1}$, while for Mg X 624-Si XII 520 the time delay of $71 \pm 6 \mathrm{~s}$ points to a wave speed of $201 \pm 17 \mathrm{~km} \mathrm{~s}^{-1}$. The sound speed in the solar atmosphere can be expressed as a function of temperature alone (Priest 1984) by the equation,

$C_{\mathrm{s}}=152 T^{1 / 2} \mathrm{~m} \mathrm{~s}^{-1}$,

where $T$ is the temperature in $\mathrm{K}$. From this and the temperatures of the lines, e.g., $2.5 \times 10^{5} \mathrm{~K}$ for $\mathrm{OV}$, we can estimate sound speeds of $76 \mathrm{~km} \mathrm{~s}^{-1}$ at the temperature of $\mathrm{OV}, 171 \mathrm{~km} \mathrm{~s}^{-1}$ at the temperature of $\mathrm{Mg}$ X 609 and 624 and $241 \mathrm{~km} \mathrm{~s}^{-1}$ at the temperature of Si XII.

The sound speed of $171 \mathrm{~km} \mathrm{~s}^{-1}$ at the temperature of $\mathrm{Mg} \mathrm{X}$ is above the speed of $154 \pm 18 \mathrm{~km} \mathrm{~s}^{-1}$ measured for the $\mathrm{OV}-\mathrm{Mg}$ X 624 line pair, suggesting that the waves detected by us from this line pair are slow magnetoacoustic in nature. However, it must be pointed out that the value of the sound speed calculated from the equation of (Priest 1984) is just an estimate that does not take into account the exact conditions of density or pressure at different heights in the atmosphere. The speed of $218 \pm 28 \mathrm{~km} \mathrm{~s}^{-1}$ measured for the O V 629-Si XII 520 line pair and the speeds of $236 \pm 19$ and $201 \pm 17 \mathrm{~km} \mathrm{~s}^{-1}$ for Mg X 624-Si XII 520 and Mg X 609-Si XII 520, respectively, are close to the sound speed of $241 \mathrm{~km} \mathrm{~s}^{-1}$ expected at the temperature of Si XII, again suggesting slow magnetoacoustic waves are responsible.

From the combination of evidence supplied from Figs. 6-11, we can therefore say that the waves causing the phase delays are propagating magnetoacoustic waves, with propagation speeds close to the sound speed. While we have seen evidence of fast magnetoacoustic waves at coronal temperatures in Fig. 10, the results from our measurements of propagation speeds suggest that these fast waves are not due to waves propagating up from 
the transition region. The fast waves may have been generated in the upper atmosphere in and around the coronal temperatures at which the Mg X and Si XII lines form.

The majority of the phase delays discussed here have been measured in locations at and above where there is known to be considerable dynamic activity, and where there has been measurements of macrospicule/blinker-like behaviour at the transition region temperature of O v, e.g., see O'Shea et al. (2005b) where datasets 26406 and 26438 are discussed. All datasets in the Northern polar region, apart from dataset 26348, show similar dynamic behaviour to these two datasets. We mention the presence of this dynamic behaviour as it is impossible to discount the possibility that it may be responsible for at least some of the slow or fast mode waves detected in this study.

Finally, we do not discount the possibility that some of the evidence for fast waves in coronal lines in Fig. 10 may be due to aliasing of much higher frequencies, above the Nyquist frequency, back into our measurable range. This aliasing is a constant and unavoidable feature of all Fourier analysis but we note that the use of the Tukey window in the Fourier techniques used has the happy effect of suppressing considerably the higher frequencies that could be aliased back into out frequency range, so reducing this possibility.

\section{Discussion and conclusions}

The vertical propagation of a sound wave in a stratified atmosphere is governed by Klein-Gordon type equations (Roberts 2004), which introduce a time-scale imposed by the equilibrium. In the case of an isothermal atmosphere, the sound speed $\left(c_{\mathrm{s}}\right)$ and the acoustic cut-off frequency $\left(\Omega_{\mathrm{ac}}\right)$ impose a natural time-scale on solutions. For a wave-front which moves away from the generation source $(z=0)$ with speed $c_{\mathrm{s}}$, the disturbance ahead of the front may be at rest, but behind the wavefront an oscillating wake is set up. The wake oscillates with a frequency $\Omega_{\mathrm{ac}}$, the natural frequency of the medium. In an isothermal atmosphere, $\Omega_{\mathrm{ac}}=\gamma g / c_{\mathrm{s}}$. In coronal conditions, a sound speed of $200 \mathrm{~km} \mathrm{~s}^{-1}$ yields an upper cut-off frequency of $0.18 \mathrm{mHz}$. Thus, waves can propagate upwards if its frequency is above this value. But one should note that this a very simplified, crude approximate model of the solar atmosphere.

Probably the first detection of propagating MHD waves in open coronal structures was made with SOHO/EIT (DeForest \& Gurman 1998). Based on the observed speeds $\left(75-150 \mathrm{~km} \mathrm{~s}^{-1}\right)$, which are close to sound speeds expected in the $171 \AA$ waveband $\left(T \approx 1.0 \mathrm{MK}, c_{\mathrm{s}}=147 \mathrm{~km} \mathrm{~s}^{-1}\right)$, and the density modulation inferred from the EUV brightness variation, it was concluded that the wave trains in plumes corresponded to slow magnetoacoustic waves, which are compressional in nature. The Alfvén waves are non-compressional and do not modulate the plasma density, in contrast to slow mode waves, while fast mode MHD waves behave somewhere in between, but generally modulate the plasma density to a lesser degree than the acoustic waves. On the other hand, both compressional slow and incompressional Alfvén waves perturb the plasma velocity, which causes the Doppler-shifts. Fast mode MHD waves have Alfvén phase speeds, which can vary over a considerable range in coronal conditions, between the minimum Alfvén speed value $v_{\mathrm{A}}$ inside of a magnetic structure and the maximum speed $v_{\mathrm{Ae}}$ outside the magnetic structure (i.e., $v_{\mathrm{A}} \leq v_{\mathrm{ph}}=\omega / k \leq v_{\mathrm{Ae}}$ ) (Edwin \& Roberts 1983). Thus, a propagating fast-mode MHD wave implies that a magnetic field disturbance travels at Alfvén speeds. The first imaging observations that have been interpreted in terms of propagating fast-mode MHD waves are the SECIS eclipse observations of Williams et al. (2002). During this eclipse, a loop has been observed with propagating wave trains in intensity, with a period of $\approx 6 \mathrm{~s}$ and a propagation speed of $2100 \mathrm{~km} \mathrm{~s}^{-1}$. More recently, Nakariakov et al. 2005) have presented evidence for fast-mode kink waves travelling at speeds of up to $700 \mathrm{~km} \mathrm{~s}^{-1}$ in a post flare supra-arcade.

In the present study, from simultaneous measurements of oscillations at transition region and coronal temperatures, evidence is found for outwardly propagating waves in off-limb polar regions. Measurements of phase delays between these oscillations provides evidence of fixed time delays between the measured transition region-coronal (O v 629-MgX 624, O v 629-Si XII 520) and coronal-coronal line pairs (Mg X 609, 624-Si XII 520). By estimating the formation heights of the different lines we estimate propagation speeds for the different line pairs that indicates that the waves producing the observed phase delays are magnetoacoustic waves propagating close to the sound speed. From the above discussion we can conclude that these waves are slow mode magnetoacoustic waves. From $I-V$ phase plots we find evidence for fast magnetoacoustic waves to be present at coronal temperatures, while at transition region temperatures slow magnetoacoustic waves are more commonly present. Finally, we find evidence, from the tendency of the phases to occur at fixed intervals of $f / 4\left(90^{\circ}\right)$ and $3 f / 16\left(67.5^{\circ}\right)$, that the observed oscillations, and, therefore, the waves that produce them, are being influenced by some form of resonant cavity through which the waves pass.

Acknowledgements. We would like to thank the full CDS team for their help in obtaining and reducing the data. CDS and EIT are part of SOHO, the Solar and Heliospheric Observatory, which is a project of international cooperation between ESA and NASA. This work was supported in part by a PRTLI research grant for Grid-enabled Computational Physics of Natural Phenomena (Cosmogrid) and PPARC grant PPA/G/S/2002/00020. We wish to thank the Royal Society and the British Council for funding visits between Armagh Observatory and the Indian Institute of Astrophysics.

\section{References}

Athay, R. G., \& White, O. R. 1979, ApJ, 229, 1147

Banerjee, D., O'Shea, E., \& Doyle, J. G. 2000, Sol. Phys., 196, 63

Banerjee, D., O'Shea, E., Doyle, J. G., \& Goossens, M. 2001a, A\&A, 377, 691 Banerjee, D., O'Shea, E., Doyle, J. G., \& Goossens, M. 2001b, A\&A, 380, L39 Bloomfield, D. S., McAteer, R. T. J., \& Lites, B. W., et al. 2004, ApJ, 617, 632 DeForest, C. E., \& Gurman, J. B. 1998, ApJ, 501, L217

Doyle, J. G., van den Oord, G. H. J., O’Shea, E., \& Banerjee, D. 1999, A\&A, 347,335

Edwin, P. M., \& Roberts, B. 1983, Sol. Phys., 88, 179

DeForest, C. E. 1998, in Proc. Int. Meeting on Solar Jets and Coronal Plumes, ed. T. D. Guyenne (Noordwijk: ESA), ESA SP-421, 63

Harrison, R. A., Sawyer, E. C., Carter, M. K., Cruise, A. M., et al.1995, Sol. Phys., 162, 233

Linnell Nemec, A. F., \& Nemec, J. M. 1985, AJ, 90, 2317

Ofman, L., Romoli, M., Poletto, G., Noci, G., \& Kohl, J. L. 1997, ApJ, 491, L111

O’Shea, E., Banerjee, D., Doyle, J. G., Fleck, B., \& Mugtagh, F. 2001, A\&A, 368,1095

O'Shea, E., Banerjee, D., \& Doyle, J. G. 2005a, A\&A, 436, L35

O'Shea, E., Banerjee, D., \& Doyle, J. G. 2005b, A\&A, 436, L43

Pauluhn, A., Rüedi, I., Solanki, S., et al.1999, Appl. Opt., 38, 7035

Priest, E. R. 1984, Solar Magnetohydrodynamics (Dordrecht, Holland: D. Reidel Publ. Co.)

Roberts, B., Edwin, P. M., \& Benz, A. O. 1984, ApJ, 279, 857

Roberts, B. 2004, ESA SP-547, SOHO, 13, 1

Rutten, R. J. 1995, Fourth SOHO Workshop Helioseismology, ESA SP-376, 151

Sterling, A. C., \& Hollweg, J. V., ApJ 1984, 285, 843

Torrence, C., \& Compo, G. P. 1998, Bull. Amer. Meteor. Soc., 79, 61; ApJ, 501, $\mathrm{L} 217$

Verwichte, E., Nakariakov, V. M., \& Cooper, F. C. 2005, A\&A, 430, L65

Williams, D. R., Mathioudakis, M., Gallagher, P., et al. 2002, MNRS, 336, 747 